\title{
Position invariant optical character recognition through symmetry features
}

DOI:

10.1109/ACT.2009.84

Link to publication record in Manchester Research Explorer

\section{Citation for published version (APA):}

Holland, S., \& Neville, R. (2009). Position invariant optical character recognition through symmetry features. In ACT 2009 - International Conference on Advances in Computing, Control and Telecommunication Technologies/ACT - Int. Conf. Adv. Comput., Control Telecommun. Technol. (pp. 313-315). IEEE. https://doi.org/10.1109/ACT.2009.84

\section{Published in:}

ACT 2009 - International Conference on Advances in Computing, Control and Telecommunication Technologies|ACT - Int. Conf. Adv. Comput., Control Telecommun. Technol.

\section{Citing this paper}

Please note that where the full-text provided on Manchester Research Explorer is the Author Accepted Manuscript or Proof version this may differ from the final Published version. If citing, it is advised that you check and use the publisher's definitive version.

\section{General rights}

Copyright and moral rights for the publications made accessible in the Research Explorer are retained by the authors and/or other copyright owners and it is a condition of accessing publications that users recognise and abide by the legal requirements associated with these rights.

\section{Takedown policy}

If you believe that this document breaches copyright please refer to the University of Manchester's Takedown Procedures [http://man.ac.uk/04Y6Bo] or contact uml.scholarlycommunications@manchester.ac.uk providing relevant details, so we can investigate your claim.

\section{OPEN ACCESS}




\title{
Position Invariant Optical Character Recognition through Symmetry Features
}

\author{
Sam Holland and Richard Neville \\ School of Computer Science \\ The University of Manchester \\ Manchester, United Kingdom \\ sam.holland@postgrad.manchester.ac.uk,r.neville@manchester.ac.uk
}

\begin{abstract}
We propose an effective method to achieve position invariance in the application of Optical Character Recognition (OCR). We normalise the position of all inputs based on their symmetry features. The Generalized Symmetry Transform (GST) is used to determine the symmetry features prior to classification by a Probabilistic Neural Network. We used the USPS data set to measure performance.
\end{abstract}

Keywords-neural, networks, probabilistic, symmetry, classification, handwritten, characters, position, invariance.

\section{INTRODUCTION}

Despite being a common feature of man-made objects, little research has been conducted on the usefulness of symmetry in pattern classification. Handwritten, offline optical character recognition performance has not yet reached that of a human [3]. For this reason, we have chosen to investigate how symmetry features can be used by connectionist systems.

This research uses the Generalized Symmetry Transform proposed by Reisfeld et al [4] to produce a continuous measure of reflectional symmetry of the patterns to be classified. An object has reflectional symmetry if it is invariant to reflection about a given plane or axis, called the reflectional plane. We've chosen to classify the patterns using a Probabilistic Neural Network based on research comparing its effectiveness to other connectionist systems [1].

In this paper we discuss our use of symmetry features to create a classifier that is invariant to changes in position. Our approach is to normalise patterns prior to presenting them to the network.

\section{Probabilistic NeURAL Networks}

Probabilistic Neural Networks (PNNs) have been shown to outperform the traditional Multi-Layer Perceptron (MLP) classifier in Optical Character Recognition (OCR) tasks [1]. Their main advantage is that their training is trivial and near instantaneous, requiring only a single pass. It is based on approximating probabilities through the use of Parzen windows [5]. They can support very complex decision surfaces which approach the Bayes optimal. The structure is depicted in Figure 1.

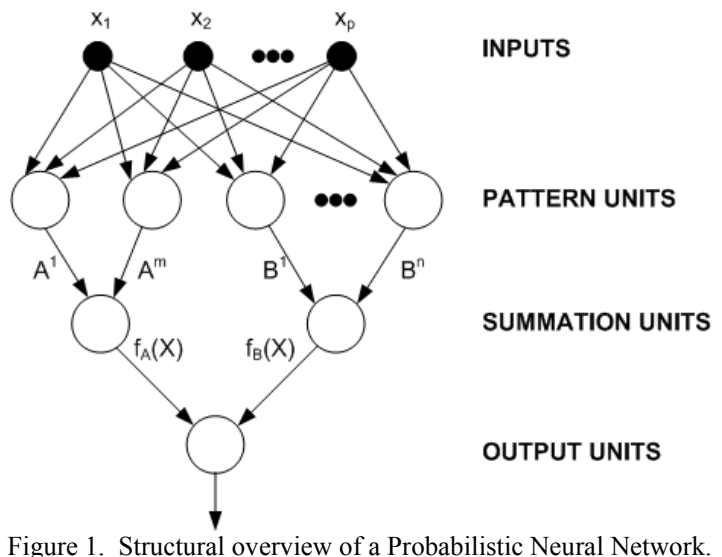

The pattern units are similar to a semi-linear neuron of an MLP except that it uses an exponential activation-output function:

$$
g\left(Z_{i}\right)=e^{\frac{Z_{i}-1}{\sigma^{2}}}
$$

A smoothing parameter $\sigma$, identical for all the units in the layer, controls the exponential scale factor. The activation, $Z_{i}$ is defined as the sum of the weighted $(W)$ inputs $(X)$ :

$$
Z_{i}=X \cdot W_{i}
$$

For each training pattern there is one pattern unit. The training pattern input is used as the weight for that pattern unit. Whilst this layer will be large for any non-trivial classification tasks, training is completed within a single pass. Recognition is slower, but training is quicker than an MLP used for the same task.

After training, the output of these units represents how similar the new pattern is to the training patterns. The unit with the highest output represents the closest matching training pattern.

The summation units simply sum the output of pattern units belonging to the same class:

$$
f_{A}(X)=\sum_{i \in A} Z_{i}
$$


The output units have two inputs and a binary output. The first input is from the summation unit of patterns belonging to that class. A summation unit of non-class members is inverted (multiplied by negative one). Hence, the activation for the output unit is:

$$
a=f_{A}(X)-f_{B}(X)
$$

The activation-output function is a hard limiter:

$$
y=\left\{\begin{array}{cc}
1 & \text { if } a \geq 0 \\
-1 & \text { if } a<0
\end{array}\right.
$$

\section{THE GENERALIZED SYMMETRY TRANSFORM}

Reisfeld et al [4] present a method for calculating symmetry magnitude and direction from the gradients of intensity in an image. The gradients are approximated using the Sobel edge operator convolution masks. For each pixel in the image, the gradient is represented by:

$$
\nabla p_{k}=\left(\frac{\delta}{\delta_{x}} p_{k}, \frac{\delta}{\delta_{y}} p_{k}\right)
$$

A vector, $v_{k}=\left(r_{k}, \theta_{k}\right)$ is associated with each pixel $k$ in the input image consisting of the magnitude of the intensity gradient, $r_{k}$; and the angle between the normal of the gradient and the horizontal axis, $\theta_{k}$.

$$
\begin{gathered}
r_{k}=\log \left(1+\left\|\nabla \mathrm{p}_{k}\right\|\right) \\
\theta_{k}=\arctan \left(\frac{\frac{\delta}{\delta_{x}} p_{k}}{\frac{\delta}{\delta_{y}} p_{k}}\right)
\end{gathered}
$$

Each pair of pixels contributes to the symmetry magnitude of a pixel mid-way between them. Hence, the set $\Gamma(p)$ consists of all the pixel-pairs that contribute to the magnitude at point $p$ :

$$
\Gamma(p)=\left\{(i, j) \mid \frac{p_{i}+p_{j}}{2}=p\right\}
$$

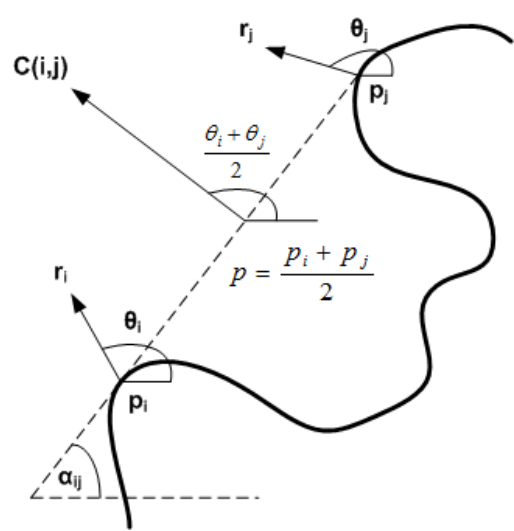

Figure 2. Two points, $i$ and $j$ contributing to the point mid-way between them.
A Gaussian-based distance weight function (controlled by $\mu$ ) favours contributions from pixel-pairs that are closer together than those further apart:

$$
D_{\mu}(i, j)=\frac{1}{\sqrt{2 \pi \mu}} e^{\left(-\frac{\left\|p_{i}+p_{j}\right\|}{2 \mu}\right)}
$$

A phase weight function favours opposing gradient orientations from a pair of pixels than those which share an orientation:

$$
P(i, j)=\left[1-\cos \left(\theta_{i}+\theta_{j}-2 \alpha_{i j}\right)\right] \times\left[1-\cos \left(\theta_{i}+\theta_{j}\right)\right]
$$

Where $\alpha_{\mathrm{ij}}$ is the angle between a line connecting points $i$ and $j$, and the horizontal axis. The total contribution of two pixel points is a function of the distance and phase weight functions:

$$
C(i, j)=D_{\mu}(i, j) P(i, j) r_{i} r_{j}
$$

The symmetry magnitude at a given point $p$ is the sum of the contributions over a set of pixel pairs, $\Gamma(p)$ :

$$
M_{\mu}(p)=\sum_{(i, j) \in \Gamma(\mathrm{p})} C(i, j)
$$

\section{POSITION NORMALISATION}

The basic idea is to reposition the contents of the pattern such that the point with the greatest symmetry magnitude is at the centre of the pattern. Where multiple symmetry axes exist, this should occur where the axes intersect. Point $s=\left(s_{x}, s_{y}\right)$ is the point where $s=\max \left(M_{\sigma}\right)$. We then apply the affine transformation:

$$
\left[\begin{array}{l}
x^{\prime} \\
y^{\prime}
\end{array}\right]=\left[\begin{array}{ll}
1 & 0 \\
0 & 1
\end{array}\right]\left[\begin{array}{l}
x \\
y
\end{array}\right]-\left[\begin{array}{l}
\left(c_{x}-s_{x}\right) \\
\left(c_{x}-s_{y}\right)
\end{array}\right]
$$

We used the point $(16,16)$ as the centre of the pattern, $\left(c_{x}, c_{y}\right)$. The affine transformation is applied to the original $32 \times 32$ pattern as opposed to the symmetry magnitude map, $\mathrm{M}_{\sigma}$. The position normalised pattern is then presented to a neural network for classification.

\section{EXPERIMENT}

The experiment used the United States Postal Service (USPS) data set. The set contains 9292 patterns each representing a handwritten digit (the numerals zero to nine inclusive.) We trained the networks with 7291 patterns. The networks' performance was measured by presenting 2001 previously unseen patterns. Based on previous research [2], the smoothing parameter, $\sigma$ was set to a value of 0.4 . This had achieved a recognition rate of $95.12 \%$.

A 16x16 greyscale image describes each digit in the original set. To test the classifier's position invariance, the input pattern's dimensionality was $32 \times 32$ pixels. The original $16 \times 16$ image was placed centrally onto the new input pattern. 
In effect, this added a padding of 8 pixels around the original data in which we could vary the position of the object. One network trained using this data. A second network trained on the output of the mechanism described in Section IV.

Fifteen additional sets of control and position normalised, training and tests sets were generated. The sets were created by moving the contents of the image in both the $\mathrm{x}$ - and $\mathrm{y}$ dimensions.

\section{RESULTS}

The optimal performance of the control classifier was $95.12 \%$. The optimal performance of the position normalised classifier was $94.67 \%$. The rate at which the performance decreases with respect to the position offset distance is depicted in Figure 3 and listed in Table 1.

\begin{tabular}{|c|c|c|c|c|}
\hline \multirow{3}{*}{$\begin{array}{c}\text { Position } \\
\text { Offset } \\
\text { Distance }\end{array}$} & \multicolumn{4}{|c|}{ Recognition Rate (\%) } \\
\hline & \multicolumn{2}{|c|}{ Control } & \multicolumn{2}{|c|}{ Position Normalised } \\
\hline & $\begin{array}{c}\text { Training } \\
\text { Set }\end{array}$ & Test Set & $\begin{array}{c}\text { Training } \\
\text { Set }\end{array}$ & Test Set \\
\hline 0 & 100.00 & 95.12 & 100.00 & 94.67 \\
\hline 1 & 30.82 & 29.70 & 100.00 & 94.67 \\
\hline 2 & 16.28 & 16.79 & 100.00 & 94.67 \\
\hline 3 & 12.30 & 13.80 & 99.52 & 94.67 \\
\hline 4 & 12.81 & 15.65 & 98.92 & 93.72 \\
\hline 5 & 16.86 & 18.59 & 97.74 & 92.68 \\
\hline 6 & 19.87 & 20.48 & 94.91 & 90.63 \\
\hline 7 & 18.46 & 19.58 & 85.90 & 80.42 \\
\hline 8 & 14.88 & 17.04 & 73.68 & 68.21 \\
\hline 9 & 15.64 & 17.34 & 61.87 & 59.59 \\
\hline 10 & 16.13 & 18.24 & 51.16 & 49.23 \\
\hline 11 & 15.84 & 18.29 & 39.80 & 37.47 \\
\hline 12 & 14.10 & 15.60 & 27.95 & 26.71 \\
\hline 13 & 11.70 & 12.31 & 19.68 & 19.23 \\
\hline 14 & 10.49 & 11.06 & 7.64 & 9.02 \\
\hline 15 & 10.04 & 9.92 & 6.62 & 7.13 \\
\hline 16 & 10.03 & 9.92 & 7.60 & 6.88 \\
\hline
\end{tabular}

Table 1. Recognition rates for the two networks, listed by data sets, against the Position Offset Distance.

\section{DISCUSSION}

Increasing the dimensionality of the input space did not affect the performance of the classifier. The control network's test set performance was equal to that in previous research. Normalising the position of the training data reduced the performance slightly, but not significantly at only a percentage-point difference of 0.45 . The control network is not tolerant of position variances. Offsetting the pattern by only one pixel in both dimensions reduces its performance to $30.8 \%$.

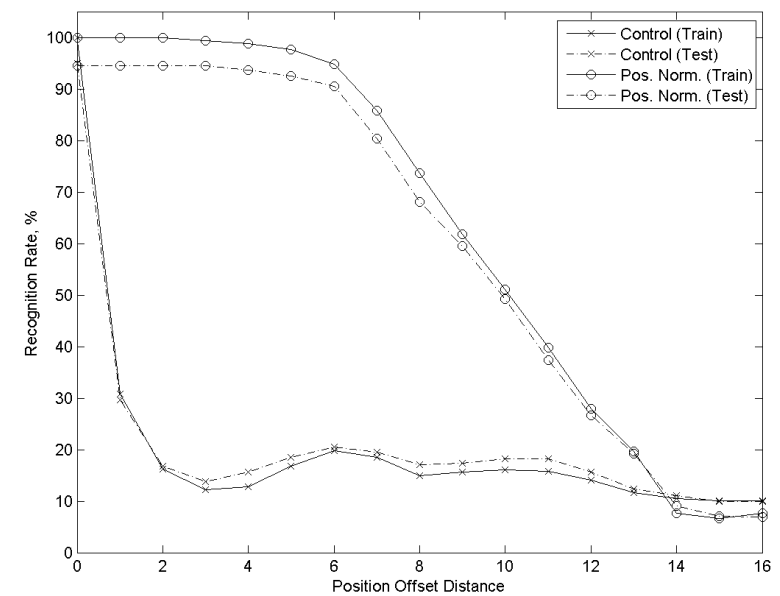

Figure 3. Graph showing the effect of shifting the object position on the recognition rate.

Using symmetry based position normalisation, the performance remains unchanged up to a distance of three pixels and then drops slightly for another three pixels. After six pixels, the rate of recognition-rate-loss with respect to position offset distance increases. We had anticipated the significant drop in performance to occur at $P=8$, when there is no 'padding' around two edges of the original data. However, this occurs one pixel early at $P=7$. It occurs one pixel earlier than predicted because of the nature of the gradient approximation method for the GST. We used convolution on only the visible portions of the pattern. The remaining reduction in the recognition rate is the result of important data no longer being visible - i.e. outside the boundaries of the image. This continues to worsen until $P=$ 16 , when none of the original data is presented to the network - only the background signal.

The results show that an affine transformation centring a pattern on the point with the highest symmetry magnitude is effective at making this classifier invariant to differences in position. The position invariant mechanism is implemented prior to classification. It should be possible to enhance other classifiers by pre-processing the input in this way.

\section{REFERENCES}

[1] D.F. Specht, "Probabilistic neural networks and general regression neural networks," in Fuzzy Logic and Neural Network Handbook, pp. 3.1-3.44, Ed, C.H. Chen, McGraw-Hill Inc, New York, 1996.

[2] S.P. Holland and R. Neville, "Classification of Handwritten Characters by their Symmetry Features", unpublished

[3] C-L Liu and H. Fujisawa, "Classification and learning methods for character recognition: advances and remaining problems," Studies in Computational Intelligence (SCI), vol 90, pp. 139-161, 2008.

[4] D. Reisfeld, H. Wolfson and Y. Yeshurun, "Context-free attentional opperators: the generalized symmetry transform," International Journal of Computer Vision, vol 14(2) pp 119-130, 1995.

[5] D.F. Specht, "Probabilistic Neural networks for classification, mapping or associative memories," Proceedings, IEEE International Conference on Neural Networks, vol 1 pp 525-532. 1988. 
